\title{
Adapting Product and Development Process for Risk Reduction in New Product Development
}

\author{
Marc Neumann, Tim Sadek, and Patrick Labenda \\ University of Bochum, Institute Product and Service Engineering, Bochum, Germany \\ \{neumann, sadek, labenda\}@lmk.rub.de
}

\begin{abstract}
The development of innovative products is characterized by uncertainties that are the result of insufficient experience and incomplete knowledge, finally leading to risks. A multitude of different methods for risk estimation is available in literature, but only scattered approaches are known that support the reduction of risks in a methodical manner. In this publication four different strategies for risk reduction are investigated that either are based on an adaption of the product or of the development process. The results of the investigation are transferred into a concept for a risk response method that is based on a risk response model and represented by using Multiple Domain Matrices. The application of the method is exemplarily demonstrated within a development project.
\end{abstract}

Keywords: Risk Management, uncertainties, Multiple-Domain Matrices.

\section{Introduction}

The development of innovative products provides business success on global markets, as it strengthens the competitive position, increases margins or offers access to new markets. However, new product development is characterized by uncertainties that are the result of insufficient experience and incomplete information and therefore endanger the compliance with budget, schedule and quality. In consequence, the development of innovative products is a necessary but also risky endeavor. In order to solve this conflict, adequate methods and effective instruments for risk management have to be established. The key to effective and efficient risk management in new product development is preventing risks instead of reacting to their impacts afterwards. This is due to the matter of fact that the impact of potential product failures increases dramatically with project progression. Strategies for risk prevention are widely known as proactive risk management [1]. Proactive risk management describes an iterative process, involving risk identification, risk evaluation and risk response. According to the described process, proactive risk management starts with the identification of uncertainties that are the trigger for potential risks. By analyzing the consequences of uncertainties and their probability to result in a failure, the risk is determined in the phase of risk evaluation. Finally, counteractions for risk reduction are identified and deployed to the project.

As the demand for proactive risk management increases, its effective application becomes challenging. Products as well as their development processes are nowadays 
characterized by an increasing level of complexity and interdisciplinarity, leading in turn to increasing complexity of the risk management process. In current practice, risk management is accomplished by bringing together all stakeholders in project meetings. The success of such project meetings depends on the presence of all stakeholders, their ability to keep the overview about all risks and their consequences and the capability to identify an ideal solution for risk response by now. It is obvious that as complexity increases, this process is not further manageable in an adequate manner. Due to the described deficits in current risk management practice, we are working on a model based risk management approach. The approach contains three partial models, denoted as "uncertainty model", "risk model" and "response model" that are directly assigned to the three phases of proactive risk management. To the best of our knowledge, no adequate methodical support for the phase of risk response is available yet. The publication at hand therefore focuses on the phase of risk response and its assigned risk response model.

\section{Fundamentals of the Uncertainty- and Risk Model}

The idea of supporting proactive risk management by a model based approach is obvious. Models are capable to reduce the inherent complexity of reality by focusing on the information of interest for a specific problem. Furthermore they are established as an adequate way for documentation of information and therefore support communication by generating a common comprehension about a situation. For detailed understanding of the presented risk response method and its assigned risk response model, it is essential to discuss the framework of the uncertainty- and risk model, first.

\subsection{Uncertainty Model}

The conceptual framework of the uncertainty model was already introduced in [2]. Assigned to the phase of risk identification, the uncertainty model delivers information about the occurrence of uncertainties within the project. Here uncertainties are defined as any condition that is not definite, known or reliable. Especially in new product development uncertainties occur throughout the entire development process due to the absence of experience. Within the uncertainty model we therefore distinguish between several types of uncertainties, denoted as "requirement uncertainties", "system uncertainties" and "process uncertainties" that lead to "quality uncertainties", "cost uncertainties" and "schedule uncertainties".

As already mentioned at the outset, product development today is characterized by increasing complexity and interdisciplinarity. Changing an initially defined requirement later on in the project, e.g., will require changes of related product elements and in turn lead to a revision of associated process steps. Here the possible change of the system and the resulting overrun of schedule and costs can in turn be interpreted as uncertainties, regarding the system and its development process. Beside the description of primary uncertainties that are the initiator for risks, the uncertainty model therefore contains information about the propagation of uncertainties throughout the 
project. To support the analyses, the model is based on the idea of supporting the investigation of uncertainty propagation by integrating structure and uncertainty analysis in a comprehensive model.

\subsection{Risk Model}

Within the risk model, the identified uncertainties are evaluated to estimate their associated risk. The measure of risk is usually defined as the product of the probability for an inadequate outcome and the impact of that outcome. Due to the generic character of common risk models, we adapted the standard risk model (e.g. [1]) to the specific context of development projects that are characterized by a high degree of novelty and complexity (see fig. 1).

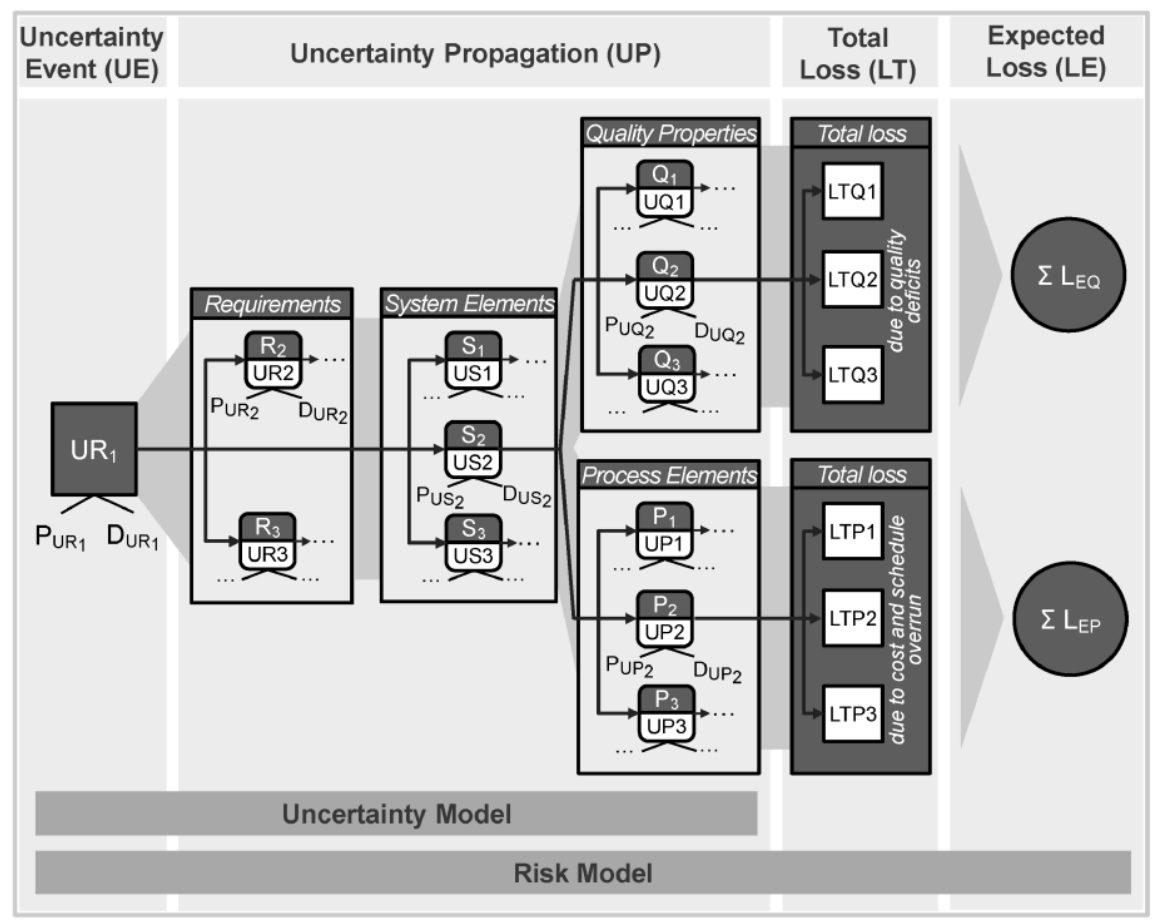

Fig. 1. Enhanced risk model for complex development projects

As depict in fig. 1 the enhanced risk model includes the uncertainty model described in 2.1. It therefore contains information about the initial uncertainty event (UE) and the propagation of uncertainties (UP) throughout the project. Additionally each uncertainty is associated with specific uncertainty drivers (D) and a probability (P) describing the likelihood of its occurrence. We differentiate between two possible paths of uncertainty propagation. The first one is directly leading to process uncertainties that are the consequence of necessary system changes (see lower path in fig. 1). The second one is leading to quality uncertainties, representing all quality deficits that 
are not corrected by additional rework (see upper path in fig. 1). Having determined the lowest level of uncertainty propagation, it is possible to assign the total losses $\left(\left(\mathrm{L}_{\mathrm{TQ}}\right)_{\mathrm{i}}\right.$ and $\left.\left(\mathrm{L}_{\mathrm{TP}}\right)_{\mathrm{k}}\right)$ to the process and quality uncertainties. The total loss is a measure for the magnitude of loss if the risk event occurs. The expected quality and process losses $\left(\mathrm{L}_{\mathrm{EQ}}\right.$ and $\left.\mathrm{L}_{\mathrm{EP}}\right)$ are calculated by multiplying the total loss by the uncertainty probabilities (see eq. 1 and 2).

$$
\begin{aligned}
\mathrm{L}_{\mathrm{EQ}} & =\mathrm{P}_{\mathrm{UE}} \times \Sigma\left[\left(\Pi \mathrm{P}_{\mathrm{UP}}\right)_{\mathrm{i}} \times\left(\mathrm{L}_{\mathrm{TQ}}\right)_{\mathrm{i}}\right] \\
\mathrm{L}_{\mathrm{EP}} & =\mathrm{P}_{\mathrm{UE}} \times \Sigma\left[\left(\Pi \mathrm{P}_{\mathrm{UP}}\right)_{\mathrm{k}} \times\left(\mathrm{L}_{\mathrm{TP}}\right)_{\mathrm{k}}\right]
\end{aligned}
$$

\section{Risk Response Strategies and Related State of the Art}

Understanding the meaning of uncertainties in new product development and the circumstances leading to risks, we are now able to identify possible strategies for risk reduction. In fig. 2 four different strategies and an extract of the underlying state of the art are given that are discussed below.

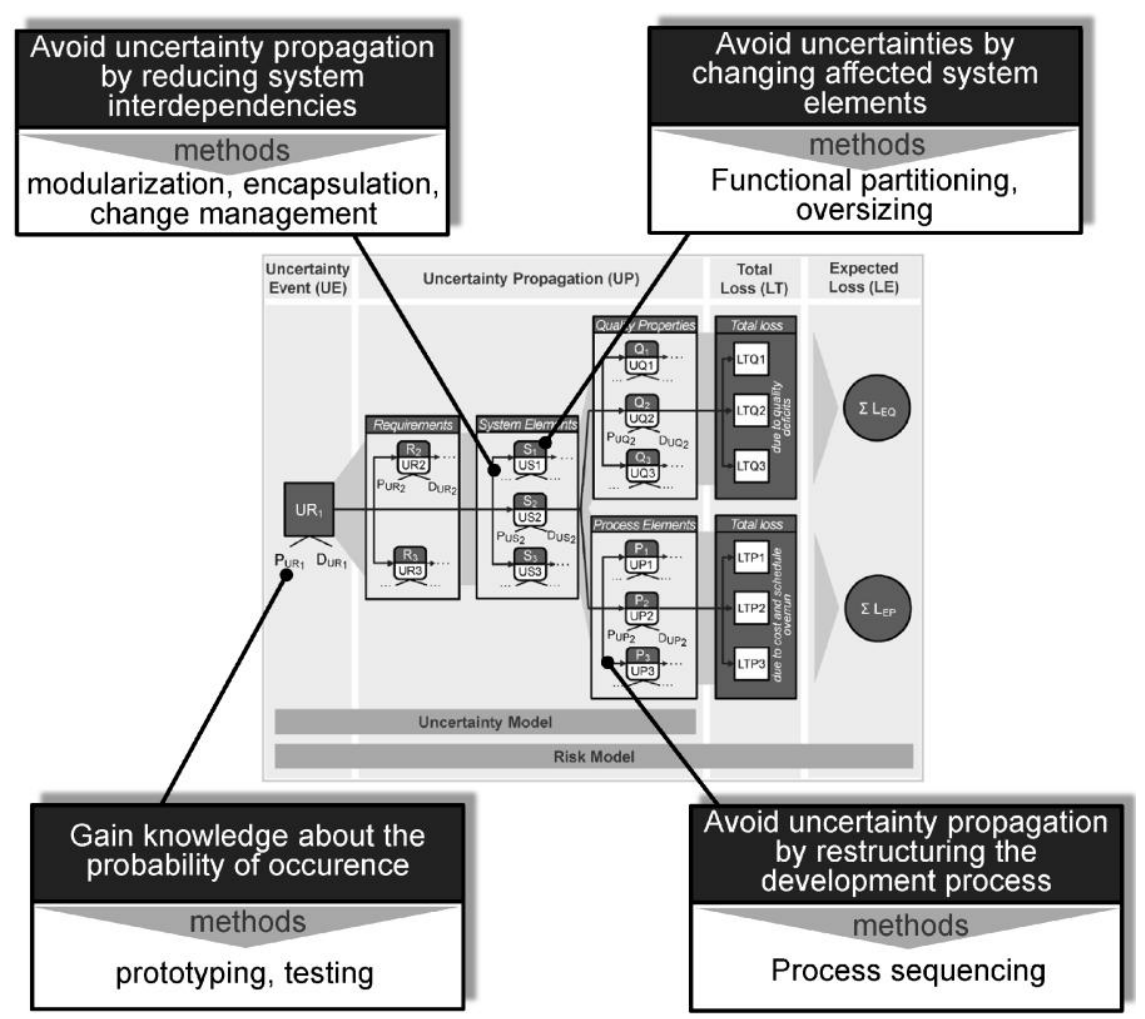

Fig. 2. Risk response strategies and associated methods 


\section{S1: Avoid Uncertainty Propagation by Reducing System Interdependencies}

As displayed within the enhanced risk model, the key impact of risks in complex development projects can be traced back to the propagation of uncertainties. Consequently, the impact of risks can be mitigated by reducing system interdependencies. Modularization describes a process of clustering system elements within modules, aiming on the reduction of interactions between these modules. Modularization can offer many benefits as it limits the impact of system changes by information-hiding [3]. Thus, modularity supports evolutionary aspects of the design process by enabling the option for future changes due to the systems' architecture. In the context of risk reduction a modular system design can be established to encapsulate uncertainties within well-defined spatial or functional system boundaries. Several useful methods and tools supporting product modularization are described in literature. The Design Structure Matrix (DSM) e.g. is a well established technique to analyze the relationship between system elements. With knowledge about the systems' interrelations, clustering routines or genetic algorithms can be applied to identify modules in the product structure [4]. Although modularization offers lots of benefits, it would be a false conclusion to believe that modularization alone offers the solution for all problems. Several disadvantages come along with modular architectures. Celona et al demonstrate that modular products are usually larger than integral products [5]. They are often heavier and less energy efficient because they are not optimized for performance. A good overview about the costs implications of product architecture differences is provided in [6].

\section{S2: Avoid Uncertainty Propagation by Restructuring the Development Process}

Similar to changing the product structure, varying the structure of the development process can affect risks in a positive manner. The development process of complex products is characterized by a multitude of highly interdependent activities that are coupled by an exchange of feedforward and feedback information. Information that are passed from one activity to another later in the sequence are called feedforward, while the ones passed back are denoted as feedback. Each activity requiring feedback information has to be accomplished on the base of uncertain assumptions, since the information is required before the downstream activity produces it. Thus, minimizing feedback loops by rearranging the process elements will reduce the risk of rework. Sequencing the development process can be supported by the use of activity-based DSMs. The analysis of activity-based DSMs can be enriched using sequencing algorithms. An overview about process sequencing and genetic algorithms is given in [7].

\section{S3: Gain Knowledge}

Traditional engineering thinking follows the philosophy of developing products topdown and sequentially. Hereby test activities are concentrated at the end of the process. Due to the obviously existing disadvantages of an absolutely sequential proceeding for new product development, iterative or evolutionary development approaches were created. These models follow the modern understanding of new product development as a learning process. Hereby uncertainties are systematically reduced by the execution of additional verification and validation activities during product development. Integrating short feedback loops into the development process 
helps testing ideas against requirements, correcting possible design errors early and therefore can help to enhance the effectivity and efficiency of the process. The adapted V-Modell used as reference for the development of mechatronic systems [8], the Plan-Do-Check-Act cycle (PDCA) postulated in the Total Quality Management (TQM) or the iterative experimentation cycle, developed by Thomke point out the same idea [9]. Experimental testing can be conducted in a multitude of different modes such as virtual prototyping, computer simulation, finite element method or rapid prototyping. Each test mode strongly differs in the necessary effort for accomplishment and the accuracy of test results. The choice of test strategy therefore significantly affects the test efficiency.

\section{S4: Avoid Uncertainties by Changing Affected System Elements}

Maybe the most obvious way of risk reduction is avoiding the occurrence of uncertainties by simply changing the affected system elements. For example, an uncertainty concerning the ability of a chosen technology to fulfill the necessary requirements can be avoided by a change of the uncertain technology itself. We denote the choice of technology as functional partitioning. In the same way system changes can guard against uncertain requirements, using design techniques like oversizing to support a robust design. Unfortunately, any change of system elements usually comes not for free. In the majority of cases the realization of "plan B" will not offer the same opportunities as the accomplishment of "plan A" does. Often just the commitment of risks leads to innovative solutions of a problem. The decision for a change therefore will result in the acceptance of opportunity costs that describe the costs for not taking demand of a possible chance.

\section{Concept of a Method Supporting Risk Response}

Four different main strategies to face risks proactively were considered in the previous chapter. They are either based on an adaption of the product or of the development process. Considering the described strategies, all of them show up specific advantages and disadvantages. Prototyping and testing e.g. support the learning process and enable the clarification of uncertainties, but additional resources have to be provided. Changing the system can reduce uncertainties indeed but can result in opportunity costs, if the change reduces the theoretically achievable quality of the system. Furthermore, each class of risk response strategy contains an almost endless number of different sub-options, e.g. virtual prototyping versus physical prototyping. They also differ strongly in the provided benefit and the necessary effort. It is obvious that additional methodical support is needed. On the following pages we therefore provide the concept of a method supporting risk response.

The framework of the risk response method is represented in fig. 3. The method is based on the response model, schematically illustrated in the lower part of fig. 3. In order to choose an adequate strategy for risk treatment, an analysis of the conceivable solution space is necessary. In general, the solution space is encompassed by the four main strategies of risk response as well as their associated sub-options. For modeling of the solution space, elements are used that we denote as response elements. Exem- 
plarily the response element test is depict in fig. 3, containing a description of the response objectives, the test object, the planned test procedure as well as a rating of the expected reliability of test results. Response requirements and restrictions are the sources of influencing factors on risk response. Furthermore, the necessary effort or required resources for each response option are essential for decision making. The response model therefore assigns context elements to the response elements that provide additional information ${ }^{1}$.

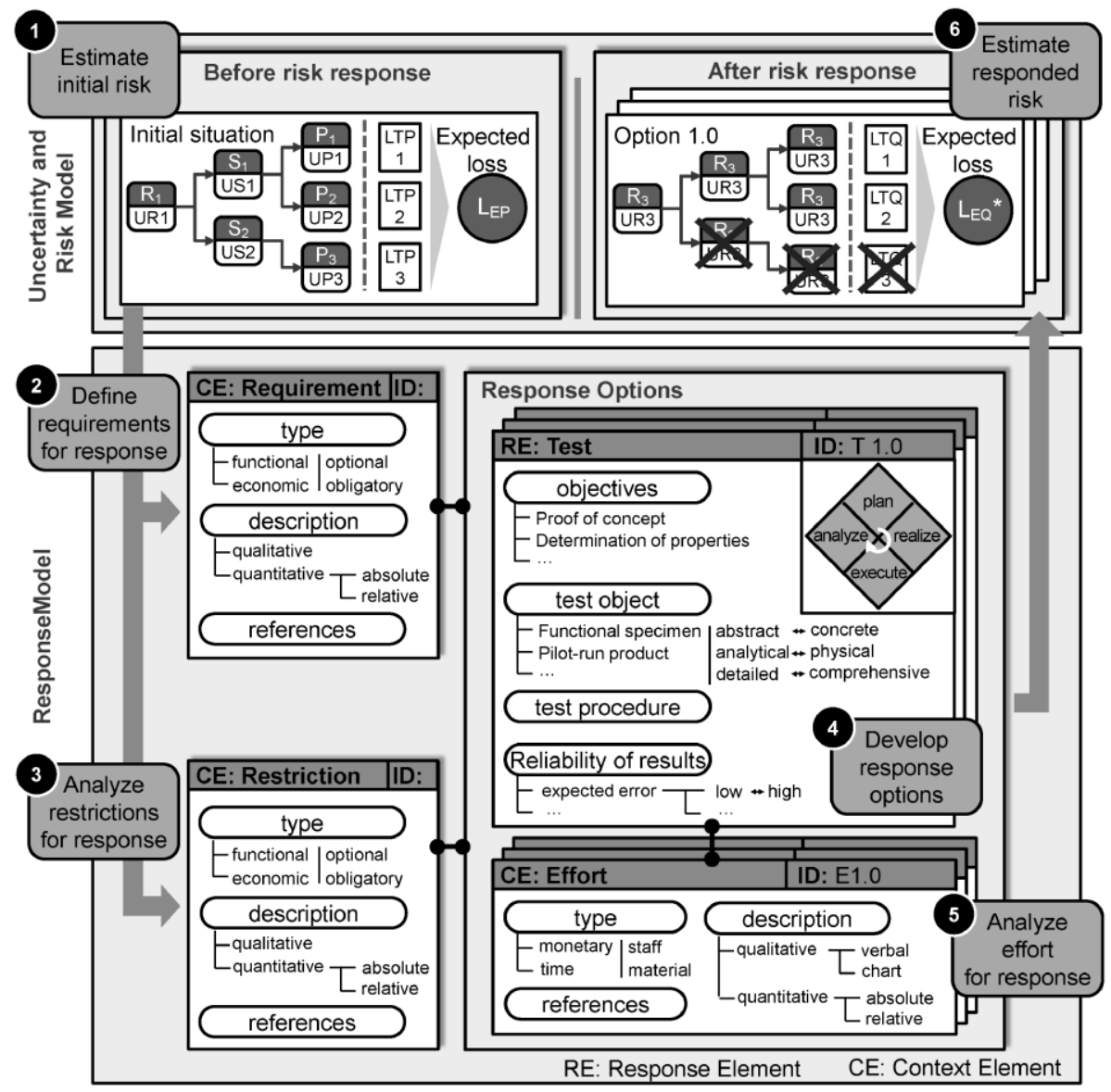

Fig. 3. Concept of the risk response method

The procedure of risk response starts with the estimation of the initial risk. The initial risk is estimated using the uncertainty model for analyzing uncertainty propagation and the risk model to calculate the expected loss. Based on the risk analysis, the requirements and restrictions for risk response are defined in the next step.

${ }^{1}$ The proposed representation scheme is based on the heterogeneous modeling approach for mechatronic systems, presented by Jansen [10]. 
Requirements can regard functional or economical aspects of risk response, e. g. the required amount of risk to be reduced or the maximal acceptable effort. Responserelated restrictions usually may derive from the aspired strategy of the company or the absence of required resources. The identified requirements and restrictions directly define the input parameters for developing adequate response options, afterwards. In order to compare the estimated response options, the necessary effort of each response option is calculated. Finally, the expected benefit is determined using the risk model again to analyze the responded risk.

\section{Exemplary Application of the Risk Response Method}

For clarification of the proposed concept, the procedure is exemplarily applied to the development of a mobile segmented robot, schematically depict in the upper part of fig. 4. Segmented robots offer a high degree of kinematic redundancy, enabling the system to adapt to the terrain. In order to maximize the tractive force by the control system, the normal force between each wheel and the ground has to be estimated. Within the exemplary application of the risk response method, we will focus on the development of the wheel module composed of the force measurement system $\mathrm{S} 1$, the drive chain S2 and the wheel S3. Essential requirements and relevant development steps are given in the table of fig. 4.

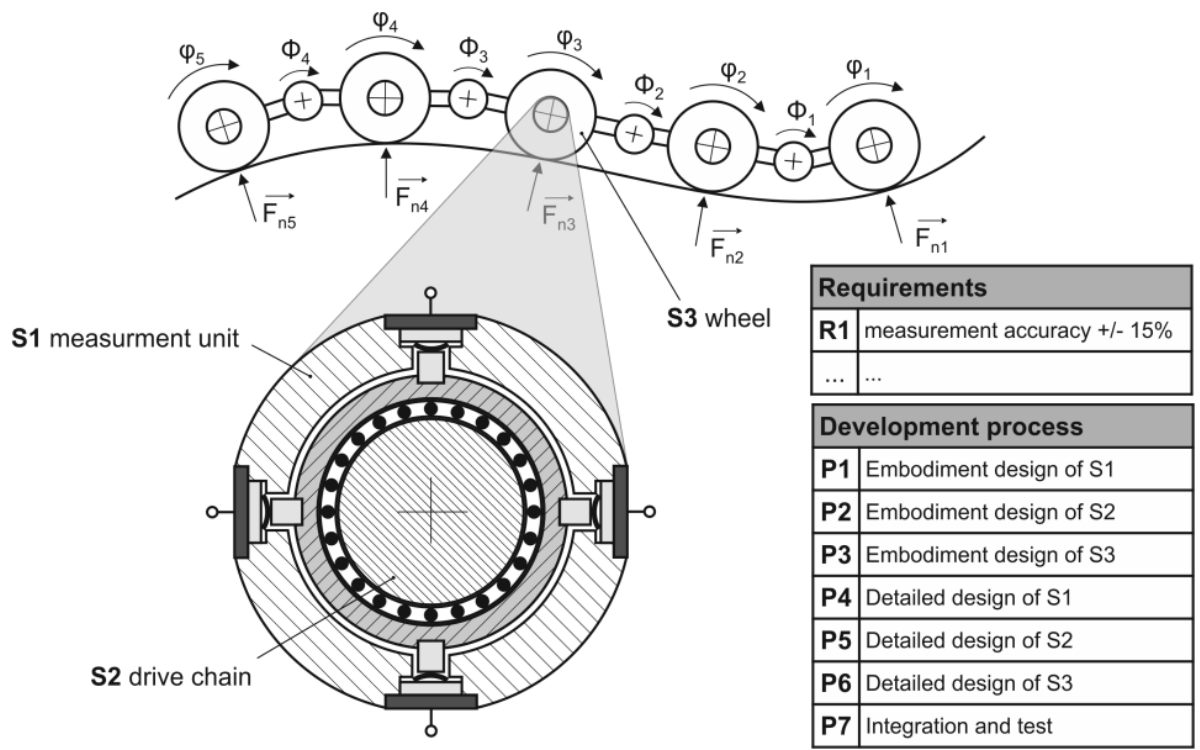

Fig. 4. Development of a segmented robot

The three system elements S1, S2 and S3 are strongly coupled by geometrical and functional interdependencies. Consequently, occurring uncertainties will propagate throughout the whole wheel unit. In order to represent the propagation of uncertainties, the uncertainty model, introduced in [2], is used. The analysis of uncertainties is 
operationalized by the use of Multiple-Domain Matrices ${ }^{2}$ (MDM), as demonstrated in fig. 5. We assume that an uncertainty regarding the required measurement accuracy exists. In consequence, the adequacy of the chosen concept for the measurement unit is called into question. The consequences of the initial requirement uncertainty on the system and its development process are visualized in the front matrix in fig. 5 (dotted line). The expected loss value is calculated by equation 2 of the risk model.

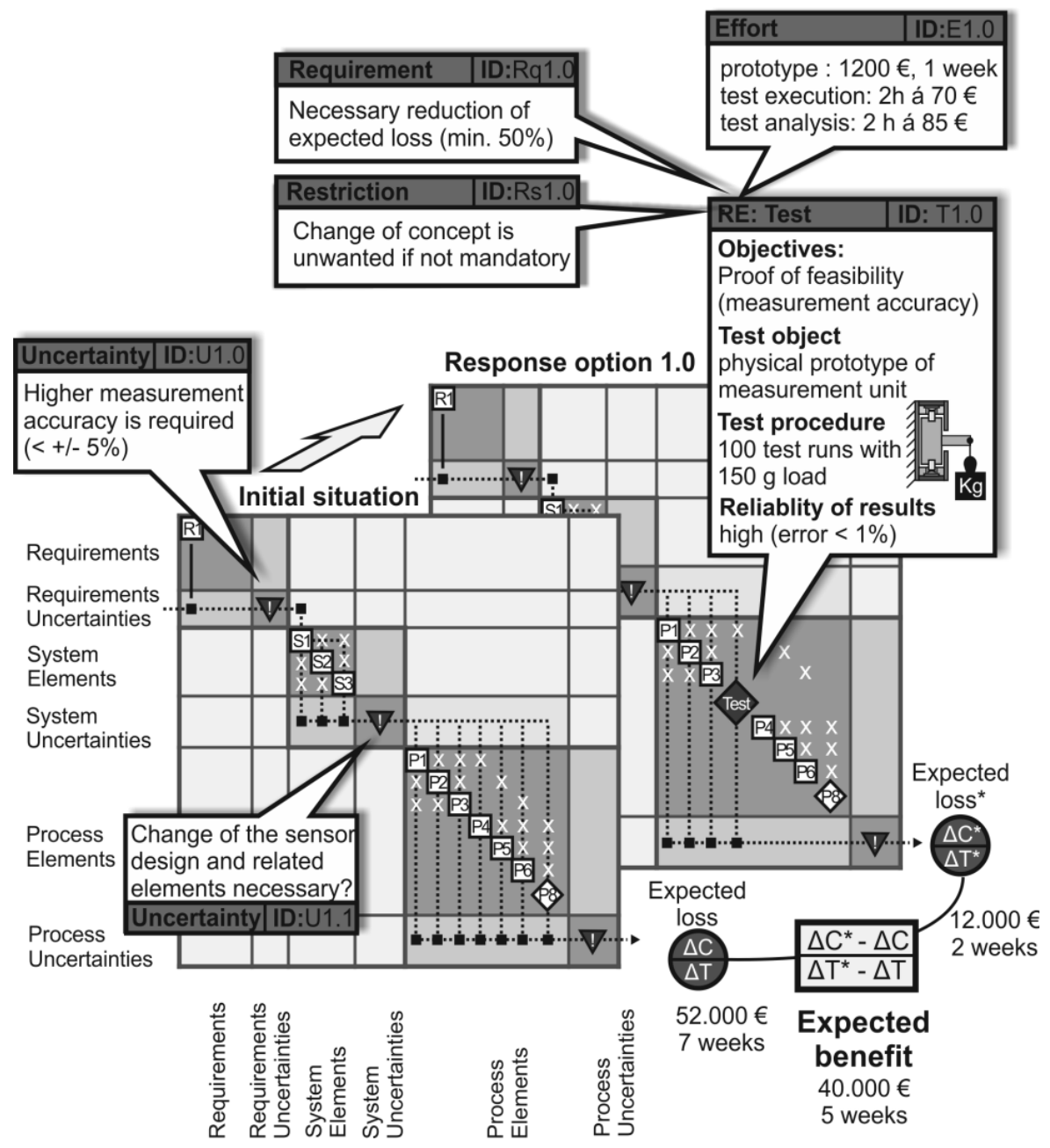

Fig. 5. Exemplary application of the risk response method

${ }^{2}$ By using MDM we are able to benefit from the advantages provided by matrices based modeling techniques (see chapter 3 ). 
Having analyzed the initial situation, requirements and restrictions for risk response are defined. In the example, a minimum reduction of the expected loss of $50 \%$ is required. The solution space is restricted by the desire, preferably not to change the system concept. Based on the given requirements and restrictions, an early test of the measurement system is proposed. As depict in the second MDM, the additional test is integrated between process steps P4 and P5, requiring the attached resources for prototyping, test execution and analysis of test result. Although the additional test apparently causes additional effort, a comparison of the expected losses shows that executing the response option will result in a remarkable benefit of $40.000 €$.

\section{Conclusion}

Treating risks proactively is the key characteristic of effective risk management in new product development. In order to reduce identified risks, adequate response options have to be generated and compared to each other. This process is not sufficiently supported by design methods and models yet. The proposed risk response method can help to overcome these deficits. Furthermore, the demonstrated application of the method within the example already indicates that practical usability of the method is strongly related to its implementation in a software tool. In future work we will therefore extend and detail the method to be transferred into a computer program.

\section{References}

1. Smith, P.G., Merritt, G.M.: Proactive Risk Management. Controlling Uncertainty in Product Development. Productivity Press, New York (2002)

2. Neumann, M., Sadek, T.: Integrating structure and uncertainty modelling using MultipleDomain-Matrices. In: Proceedings of the International Design Conference (DESIGN 2012), Dubrovnik, Croatia (submitted for review, 2012)

3. Parnas, D.L.: On the criteria to be used in decomposing systems into modules. Communications of the Association for Computing Machinery 14 (1972)

4. Rogers, J., Korte, J.J., Bilardo, V.J.: Development of a Genetic Algorithm to Automate Clustering of a Dependency Structure Matrix, NASA Center for AeroSpace Information, Paper No. NASA/TM-2006-214279 (2006)

5. Celona, T., Embry-Pelrine, C., Hölttä-Otto, K.: Are modular products larger than integral products? In: Proceedings of the International Conference on Engineering Design (ICED 2007), Paris, France (2007)

6. Fixson, S.K.: Linking Modularity and Cost: A Methodology to Assess Cost Implications of Product Architecture Differences to Support Product Design. MIT (2002)

7. Browning, T.R.: Applying the Design Structure Matrix to System Decomposition and Integration Problems: A Review and New Directions. Transactions of Engineering Management 48(3) (2001)

8. VDI: VDI-Richtlinie 2206: Entwicklungsmethodik für mechatronische Systeme. Beuth Verlag, Berlin (2004)

9. Thomke, S.H.: The economics of experimentation in the design of new products and processes. MIT (1995)

10. Jansen, S.: Eine Methodik zur modellbasierten Partitionierung mechatronischer Systeme. Shaker Verlag, Aachen (2007) 